\title{
Y Rizoma \\ Circulación / circulaciones en la investigación en plataformas mediáticas
}

Resumen: La investigación sobre plataformas multimediáticas José Luis Fernández va ampliando el horizonte sobre la circulación de los discursos mediatizados. Tienden a verse como procesos de circulación, tanto fenómenos micro, como fenómenos macro, procesos que se presentan en un solo espacio mediático o que atraviesan diversos medios, incluso relaciones entre intercambios cara a cara y mediatizados. En este artículo, se presenta el estado actual del trabajo del equipo sobre esos procesos de circulación.

Palabras clave: plataformas, mediatizaciones, discursividades, circulación.

\section{Circulação / circulação na pesquisa em plataformas de mídia}

Resumo: A pesquisa em plataformas multimídia está expandindo o horizonte da circulação de discursos mediatizados. Eles tendem a ser vistos como processos de circulação, ambos os micro-fenômenos e macro fenômenos. Processos que se apresentam em um único espaço de mídia ou que passam por diferentes mídias. Até mesmo relações entre trocas cara-a-cara e mediatizadas. Neste artigo, é apresentado o estado atual do trabalho da equipe nesses processos de circulação.

Palavras-chave: plataformas, mediatizações, discursividades, circulação

\section{Circulation / circulation in research on media platforms}

Abstract: Research on multimedia platforms is expanding the horizon on the circulation of mediated discourses. They tend to be seen as processes of circulation, both micro phenomena, and macro 

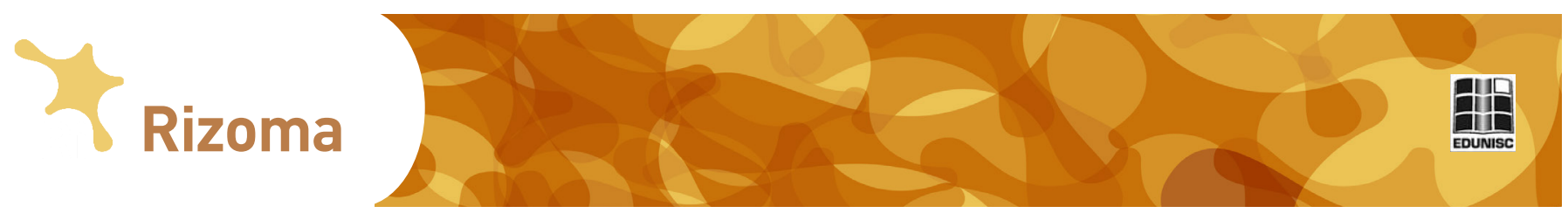

phenomena, processes that occur in a single media space or that go through different media, including relationships between face-to-face and mediated exchanges. In this article, the current state of the team's work on these circulation processes is presented.

Keywords: Plataforms. Mediatizations. Discursiveness. Circulation.

\section{Introducción}

En este artículo se propone continuar reordenando nuestro campo de investigación sobre los intercambios discursivos en plataformas mediáticas, diferenciándolo, como objeto de estudio, de otros fenómenos con los que convive.

Se propondrá derivar hacia la problemática de la circulación a diversos rasgos y niveles que conviven con los intercambios discursivos mediáticos. Es un gesto tal vez demasiado audaz, pero justificado por el desarrollo de investigaciones que obligan a dar respuestas a propuestas teóricas con las que se intersecta.

Para la investigación desde la semiótica de las mediatizaciones, la noción de circulación nunca fue una noción de diccionario; y menos de enciclopedia. Las teorías de los efectos de las mediatizaciones de origen no semiótico, todavía se asientan en la confianza de que, lo que ocurre entre un texto y sus receptores se puede comprender, tanto evaluando los contenidos mediáticos como preguntando a los receptores acerca de qué recuerdan y/o comprendieron en su recepción: se trataría del mismo campo fenoménico. Eliseo Verón rompió con ese sentido común de los estudios sobre la cultura masiva, y propuso considerar a la circulación como campo diferencial entre producción y reconocimiento (VERON, 1987: 126-133).

En la actualidad, los estudios sobre circulación están en expansión entre quienes venían trabajando con Verón, en el proceso de adecuación de esa propuesta a las nuevas realidades en las mediatizaciones (Neto, 2010; Traversa, 2016; Carlón, 2016, 2018; Cingolani, 2017). Desde ese punto de vista, se propone a la circulación como un fenómeno central en la comprensión de esta etapa de las mediatizaciones postmasivas. En ese camino, este artículo pretende, como mucho, ser un aporte lateral a esos avances.

El problema es que es en el estudio de las nuevas mediatizaciones convergen diversos niveles $\mathrm{y}$, más precisamente aún, prácticas, entre las cuales es difícil establecer campos de coincidencia. Ello obliga a que los avances tengan en cuenta otros puntos de vista teóricos y otras maneras de insertarse en el proceso de mediatización con las que se intersecta. Al investigar plataformas mediáticas, por ejemplo, se construyen vínculos con diseñadores de entornos de usuario, o de 


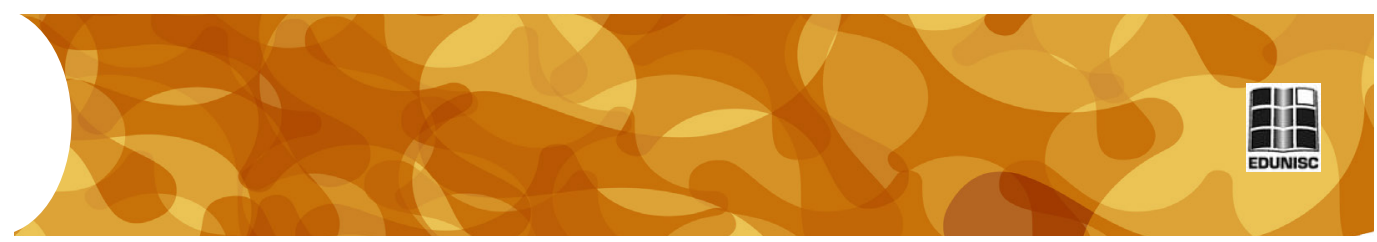

interfaces y aplicaciones, con programadores y teorizadores sobre el peso del software, y con resultados producidos por las plataformas y sus redes, como los habitualmente denominados de big-data (FERNÁNDEZ, 2016b; 2018). Ciertos aspectos que se consideran como propios del gran universo de la circulación mediática aparecen como muy cercanos al estudio de los intercambios discursivos en plataformas, y hasta formando parte de él; otros, en cambio, parecen más alejados de la superficie de las interfaces; pero con ambos tipos se convive y se interactúa.

La importancia novedosa de la circulación en el universo de la mediatización se soporta en la disolución de las distancias entre producción y reconocimiento, propias de los medios masivos:

\footnotetext{
"A circulação ao deixar de ser uma problemática de intervalos entre elementos de um determinado processo de comunicação, passa a se constituir em um dispositivo central, uma vez que as possibilidades e a qualidade das interações sóciodiscursivas se organizam cada vez mais em decorrência da natureza do seu trabalho em dar forma à arquitetura de procesos comunicacionais. As lógicas dos "contratos" são subsumidas por outras "lógicas de interfaces" (FAUSTO NETO, 2010: 12).
}

El pasaje de la lógica de los contratos de lectura a la lógica de las interfaces sería la gran transformación de las arquitecturas de los procesos comunicacionales. Es muy importante también aquí, la aparición con fuerza de la noción de interacciones sociodiscursivas como núcleo central de lo que habría que describir y explicar.

Al revisar, desde el punto de vista de las comparaciones entre enunciación e interacción, el concepto ya citado de circulación como diferencial entre producción y reconocimiento, y a la luz de nuevos conocimientos, se sostuvo que hay convivencia entre las lógicas de los contratos y las lógicas de las interfaces. Para no repetir todo el análisis, pero para facilitar la comprensión de lo que se expondrá aquí, se tuvo en cuenta la necesidad de no abandonar aquella diferenciación porque:

Para decirlo con precisión, cada posteo en un muro de Facebook, cada tweet, cada publicación en YouTube, puede generar diversos reconocimientos, así como cualquier mensaje telefónico, televisivo o gráfico. La diferencia entre previas y nuevas mediatizaciones no puede estar allí, porque ambas se encuentran inscriptas en diversos entramados discursivos y culturales que generan diferentes gramáticas de reconocimiento (FERNÁNDEZ, 2016: 20-21).

Es decir, que debía aceptarse el encontrar la convivencia de ambos modelos. Y ello llevó, por decirlo así, a ablandar el concepto veroniano de circulación, y su corrección por Fausto Neto, para hacer frente a los diversos fenómenos circulatorios a descubrir. Nuestro aporte aquí será el de realizar una revisión del campo que encontramos como propio de la circulación, desde el punto de vista de 


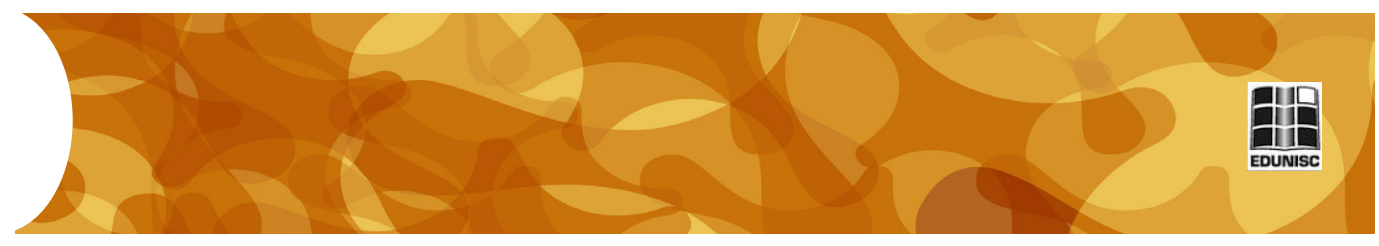

nuestro desarrollo de investigación. Es coincidente esta perspectiva con la autocrítica de la noción de circuito, propuesta por Jose Luis Braga y revisada desde el punto de vista de su investigación (BRAGA, 2012: 48-51). Sin embargo, como se verá, volveremos a ella cuando el análisis lo haga necesario.

Entre los que ven a la circulación como un fenómeno clave para comprender las mediatizaciones en la hipermediática época contemporánea, está Mario Carlón. En un artículo reciente, en el que aborda la desafiante presencia de lo individual en las redes, ha recuperado de trabajos previos cuatro modelos temporales, pero también espaciales, sobre los que sigue trabajando, para capturar las diversas posibilidades de circulación entre medios masivos y redes: ascendentes, descendentes, horizontales $\mathrm{y}$, ahora, transversales. (CARLÓN, 2018: nota $4 \mathrm{~s} / \mathrm{p}$ ).

Entre otros fenómenos recorreremos aquí aspectos de esa visión general sobre la circulación en sentido estricto, pero veremos diversos fenómenos micro que recrean o cuestionan esas clasificaciones. La hipótesis a explorar es que resulta difícil que un concepto tan amplio como el de circulación se adapte a todos los emergentes que se le puedan atribuir.

El objetivo será, desde ese punto de vista, más superficial y comparativo que profundizador: del concepto macro de circulación, hacer observaciones de distintos aspectos desde la experiencia en el estudio del fenómeno. En primer lugar, se situará la problemática de la circulación en el estado actual de la investigación sobre plataformas mediáticas y se procurará mostrar que esa posición tiene utilidad; luego se presentarán casos que pueden considerar como propios de la circulación en plataformas; se las relacionará con aproximaciones que se observan como cercanas y complementarias al universo de lo discursivo mediático.

\section{La circulación entre otros fenómenos a estudiar en plataformas mediáticas}

En primer lugar, se presenta el camino de trabajo realizado sobre los intercambios mediáticos, representado en dos esquemas. En la Figura 1, se esquematiza cómo se constituye el objeto de estudio sobre los discursos de los medios masivos desde una perspectiva sociosemiótica.

Descripto superficialmente, la sociosemiótica, por un lado, estudia los discursos en producción, es decir las emisiones y, por otro lado, la etnografía, o los estudios cuantitativos, estudian a la audiencia por el otro, más allá de que desde la sociosemiótica se considere al 


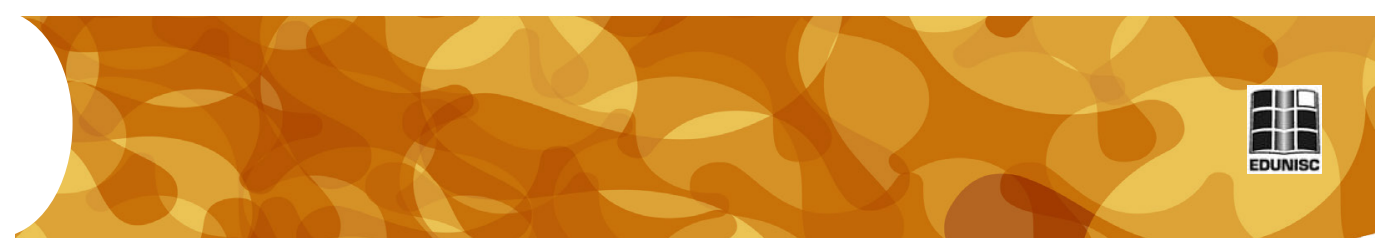

análisis semiótico como insumo clave para el trabajo de las disciplinas que estudian los efectos (FERNÁNDEZ, 2012).

Figura 1 - Sociosemiótica y medios masivos

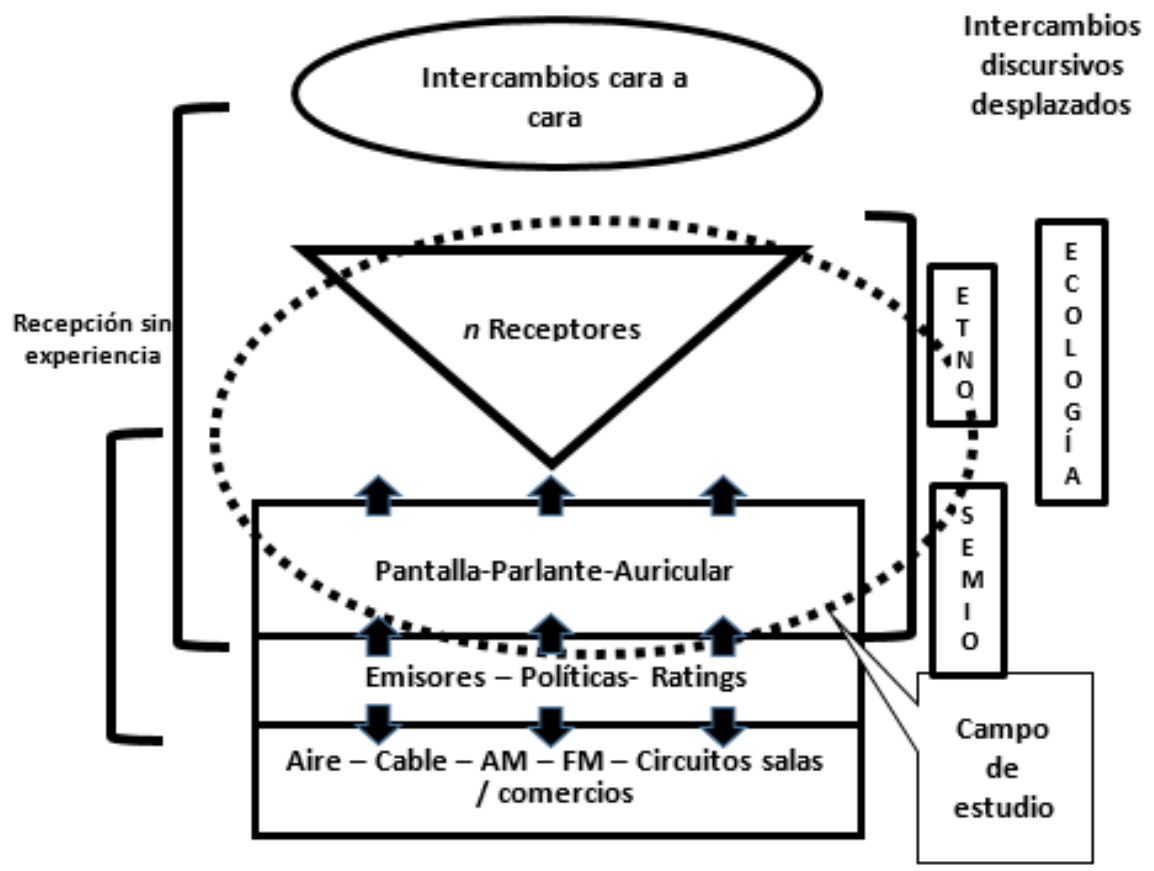

Allí se ve que entre producción y reconocimiento se presenta la distancia veroniana, sea temporal, espacial o espacio-temporal, que los receptores de los medios no participan de la experiencia de emisión y que hay distancias también entre los estudios discursivos y los denominamos aquí como etnográficos. Algo muy importante de este sistema, denominado genéricamente como broadcasting es que los receptores, pocos o muchos, son en principio, desconocidos para los emisores: cada emisión es una búsqueda incierta de receptores. De ellos sólo se tendrá respuestas minoritarias y siempre desplazadas, espacial o temporalmente. El rating, con sus limitaciones, fue el gran descriptor de la indeterminada audiencia televisiva.

Lo que ocurre con las nuevas mediatizaciones, sus plataformas y sus redes, se representa en la Figura 2.

Como se ve allí, el nuevo campo de trabajo debe incluir la presencia de los que, frente a un posteo (emisión) tienen la posibilidad de responder, es decir intervenir, al menos en una primera mirada en el mismo campo, interfaz, en el que se produce la emisión (FAUSTO NETO, 2010). Este cambio es el que se ha considerado, con razón, como fundacional de una nueva etapa de las mediatizaciones. 


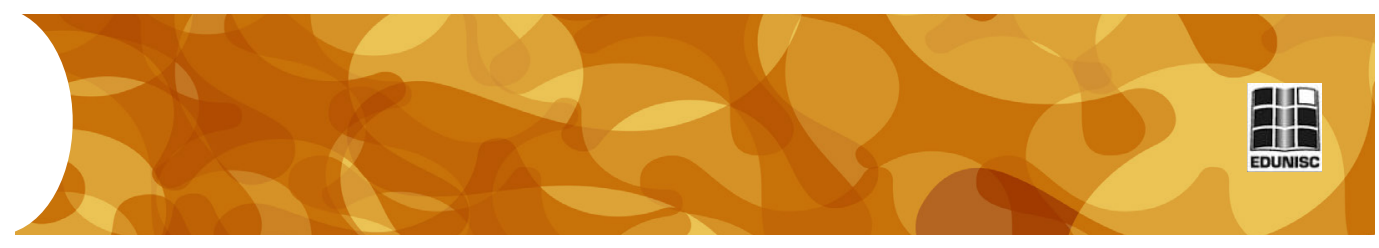

Figura 2 - Sociosemiótica + Etnografía de las plataformas

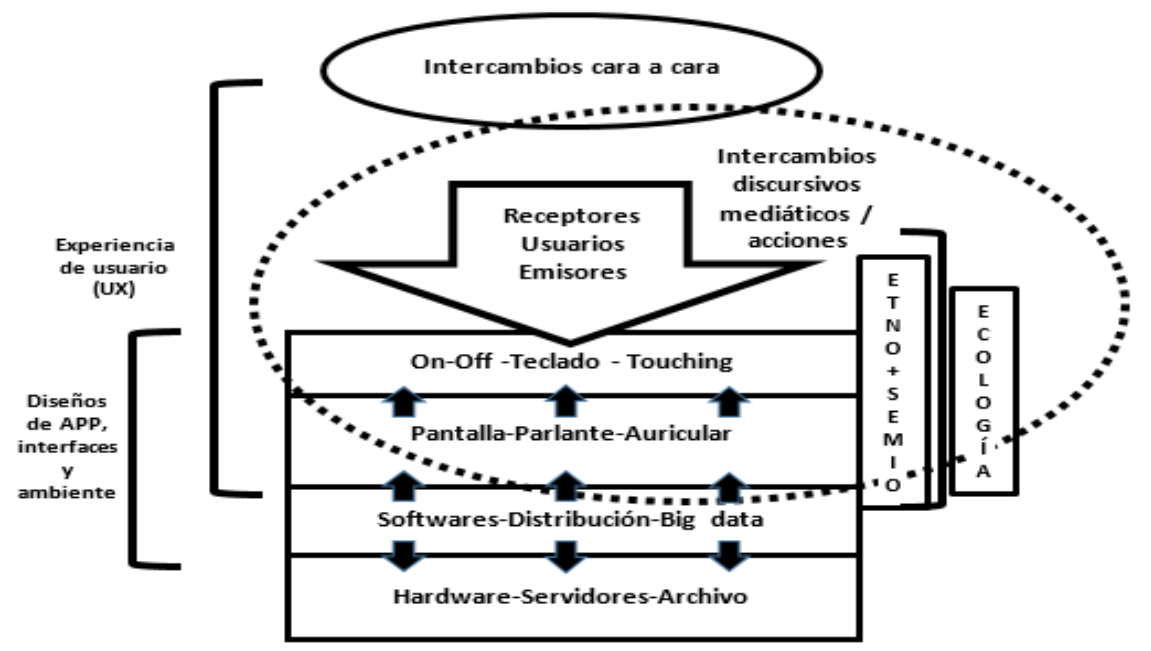

En la Figura 1 se advierte el efecto de intervalo que describe Fausto Neto, y en la Figura 2, la aparición de la lógica de las interfaces: la circulación desde este punto de vista macro se ha transformado. En ambas figuras quedan afuera del campo de interés de esta línea de reflexión, lo que se ha denominado como "sub-discursivo" (CINGOLANI, 2016): las materialidades técnicas, los modos de emisión o recepción, los sistemas de software o hardware.

Se dejan de lado aquí todos los otros cambios entre los dos modelos, pero se anotan la necesaria aproximación entre las metodologías semióticas y las etnográficas (megustear, compartir, subir, son acciones, no sólo operaciones discursivas), y el cambio en la cercanía e importancia de un punto de vista ecológico para comprender qué es lo que está ocurriendo en estos intercambios en interfaz.

En términos generales, en este artículo, se citarán trabajos cercanos y se solicita disculpas por ello. No resulta fácil trabajar desde y sobre working in progress y hasta es difícil establecer las referencias bibliográficas. A partir de aquí, presentamos diversos sistemas de intercambio y de circulación.

\section{Circulaciones intrapantalla}

Como se verá, la circunscripción al espacio pantalla tiene sus problemas. Como dice Gastón Cingolani “...El tamaño de una página puede ser muy variable, y el escroleo (o scrolling) es la acción que permite traspasar esa frontera y recorrer el largo y ancho de la misma..." (CINGOLANI. 2016: s/p). Aquí no tensaremos los límites de la pantalla, veremos que, cuando pasemos a extensiones que la hacen poner en cuestión, hablaremos ya de circulaciones intraplataformas 


\section{$\gamma_{\text {Rizoma }}$}

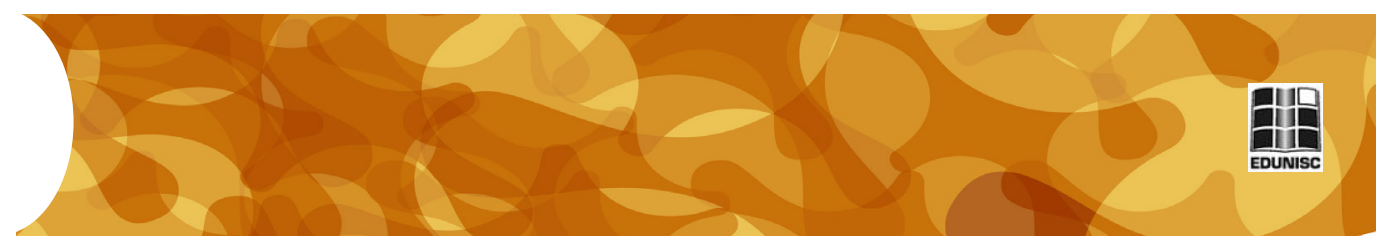

Vamos a presentar sólo un ejemplo que muestra la complejidad de las interacciones discursivas en una plataforma de uso cotidiano como Facebook y cuyo análisis más profundo hemos realizado en otro lugar (FERNÁNDEZ, 2018: 101-110). En la Figura 3 se ve un breve intercambio producido en un muro de FB (A) y en el que intervienen otros dos usuarios, a los que hemos denominado B y C. Si bien no llega a leerse lo escrito, es importante ver el cotexto/contexto en el que se produce el intercambio: en Facebook es muy difícil de sostener el efecto de transparencia de la mediatización.

Se puede ver en la figura que el intercambio ocupa un lugar central, pero en competencia con el conjunto de la pantalla de Facebook. Se presentan aquí los posteos escriturales con breves anotaciones extraídas del texto citado.

Figura 3 - Muro de A en Facebook

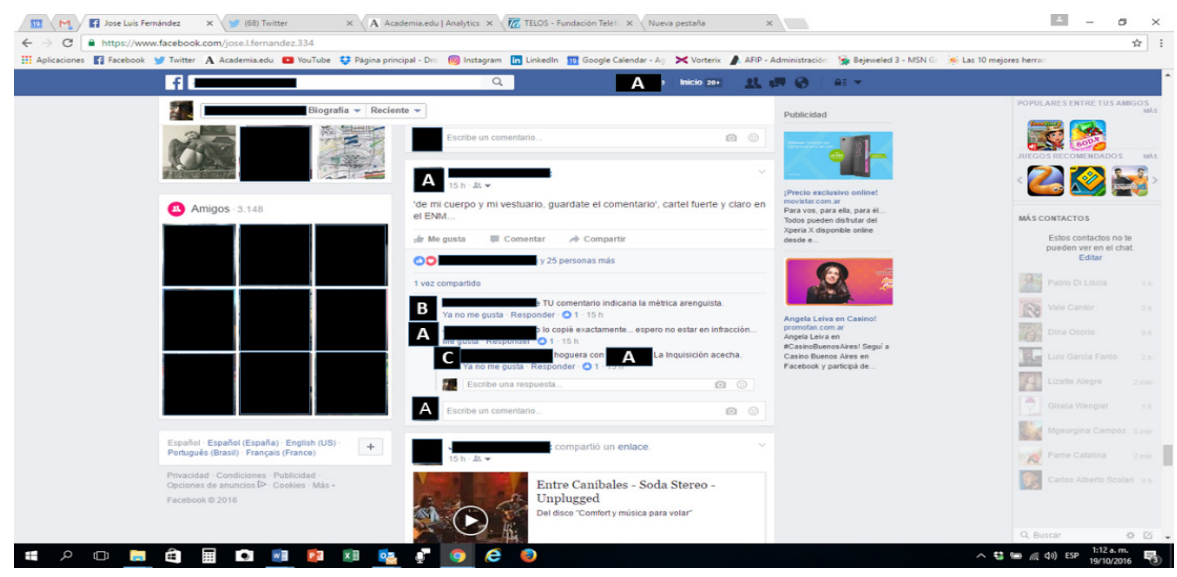

A. 'de mi cuerpo y mi vestuario, guardate el comentario', cartel fuerte $y$ claro en el ENM... En primer lugar, se registra la referencia a un cartel y al Encuentro Nacional de las Mujeres en la ciudad Rosario, Argentina. Es decir que este posteo, publicado en una plataforma global, aunque sólo para amigos de Facebook (una posibilidad más restringida que Público).

B. Guardate TU comentario indicaría la métrica arenguista. Este primer comment hace referencia en tono juguetón a cómo debería adaptarse el slogan reproducido a la oralidad de la consigna cantada, si fuera cantada.

A. no lo copié exactamente... espero no estar en infracción... Esta respuesta cambia la escena: se introduce una disculpa sobre la imprecisión posible de la cita y aparece también el riesgo de la sanción frente a un error;

C. A la hoguera con A.! La Inquisición acecha. La intervención de C., como se ve, profundiza el toque irónico de A. 
Esto se ve algo atenuado porque la respuesta no es en el nivel de la secuencia general sino, como permite desde hace un tiempo Facebook, responde a A. sin incluir necesariamente a $\mathrm{B}$.

A pesar de la brevedad del ejemplo y la superficialidad de las observaciones, el análisis obligará a revisar constantemente al menos cuatro capas superpuestas de contextos:

- la de la secuencia que, cuanto más extensa es, más obliga a controlar la linealidad o la ramificación de la misma;

- las relaciones con el conjunto del muro;

- las referencias al contexto social directo

- el universo de gramáticas de reconocimiento requerido para que el intercambio, primero, exista y, luego, se pueda comprender su campo conflictivo.

Como se ve, este ejemplo sencillo ya obliga a la convivencia entre las lógicas de contrato y las lógicas de interfaces.

\section{La circulación intraplataformas. Secuencias vs estados.}

Comencemos la deriva entre lo que consideramos como fenómenos de circulación con un caso de gran interés, teórico y social, pero de relativa simplicidad espacial: el de los comments que suceden a diversos tipos de publicación.

Un clásico ya de las plataformas informativas on-line es que, ante la publicación de una noticia (en general, más que un posteo), se desate una catarata de comments que suelen sorprender por su virulencia. Es el imperio de los haters, usuarios que descargan muchas veces su ira, o su acidez, sea sobre aspectos de la publicación original, sea sobre otros comentadores. Ello obliga muchas veces a los administradores al cierre del espacio de comments o a la edición sobre la marcha, procedimiento que adquiere una relevancia y una dificultad mucho mayores en el live blogging, en la medida en que en esto tipo de textos tanto el periodista como el lector producen su discurso en el presente de la enunciación (THURMAN y WALTERS, 2013; PORTO LÓPEZ, 2014).

Es verdad que, en un extremo, algunos posteos en Facebook o en Twitter que generan muchos comments, hace que el desarrollo de éstos genere ramificaciones y conflictivades laterales, pero, al menos por ahora, parece cómodo diferenciar estos casos en que, hay una publicación aislable, y sus efectos escriturales de respuesta.

Pablo Porto López se ha dedicado a temas de edición on-line. Sus publicaciones comienzan con un caso de mediatización transmedia: el modo en que la prensa ofrece cubrir on-line eventos deportivos (PORTO LÓPEZ, 2010) y finaliza con su Tesis de Maestría en Análisis 


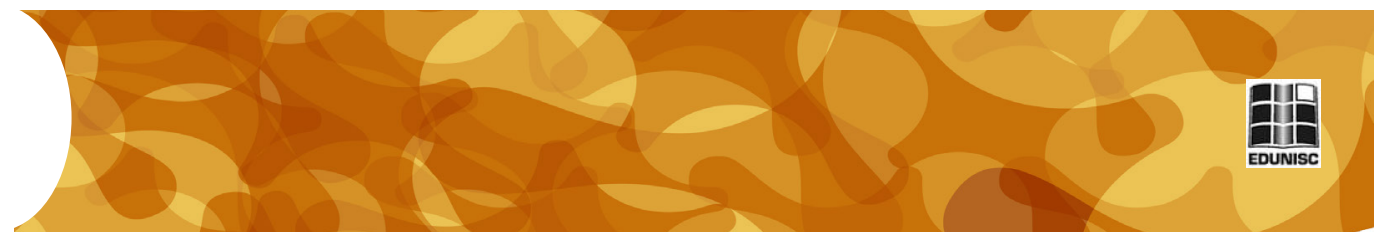

del Discurso (PORTO LÓPEZ, 2018). Allí Porto López genera una importante descripción de los alcances y los límites de la interacción escritural en nuevos espacios de mediatización, las diferencias, claves para el periodismo on-line entre la crónica simultánea y la crónica retrospectiva, y finaliza reflexionando sobre la presencia del estilo informal en la escritura y la dimensión clave de lo pasional.

No podemos citar aquí, por falta de espacio, todos los trabajos que analizan secuencia de publicaciones sobre un tema en Facebook o en Twitter, sea en términos de análisis discursivos, o de las métricas que los monitorean. Pero no siempre esos trabajos consiguen comprender el fenómeno de circulación en las plataformas, porque una secuencia de comentarios se puede producir en cualquier mediatización que lo permita materialmente. La pregunta a responder en términos de circulación mediática específica es: ¿una secuencia idéntica de comentarios publicada en Facebook y en Twitter, es la misma circulación mediática?

Por otra parte, el estudio de secuencias de comments en las diferentes plataformas convive con estudios que tratan de reconstruir estados de opinión. Un caso sobre el que van publicándose diversos tipos de estudios es acerca del análisis del conjunto de posteos que se etiquetan a través de un hashtag. El hashtag permite la convergencia de diferentes publicaciones, muy disímiles entre sí, pero que abordan un tema (las dimensiones y características de esta noción de tema debería ser estudiada muy pormenorizadamente desde la semiótica y aún desde los análisis de contenidos).

El hashtag, y los etiquetados en general, son componentes importantes de los procesos de circulación en las plataformas y sus redes $\mathrm{y}$, sin embargo, parece que todavía no han generado investigaciones $\mathrm{y}$ teorías, aunque es posible que muy pronto comiencen a aparecer sus resultados. Lo que sí, hay progresivas publicaciones presentando resultados a partir de las distribuciones de posteos que se organizan alrededor de algunos ellos.

Natalia Aruguete y Ernesto Calvo han publicado varios trabajos con esa orientación, entre ellos alrededor del haschtag \#Maldonado, una etiqueta con gran repercusión y controversias. En el artículo, además de reflexiones acerca de los trolls, muestran resultados que diferencian usuarios de derecha o de izquierda y, a pesar de utilizar diversas metodologías cuantitativas de análisis, el resultado final es algo descorazonador:

De allí que la información se propague, no por su veracidad ni por la codicia maquiavélica de los líderes políticos, sino porque circula en una región donde sus moradores se encuentran sensibilizados para interpretar el mundo desde tales comunidades, cerradas y polarizadas (Aruguete y Calvo, 2018: s/p). 


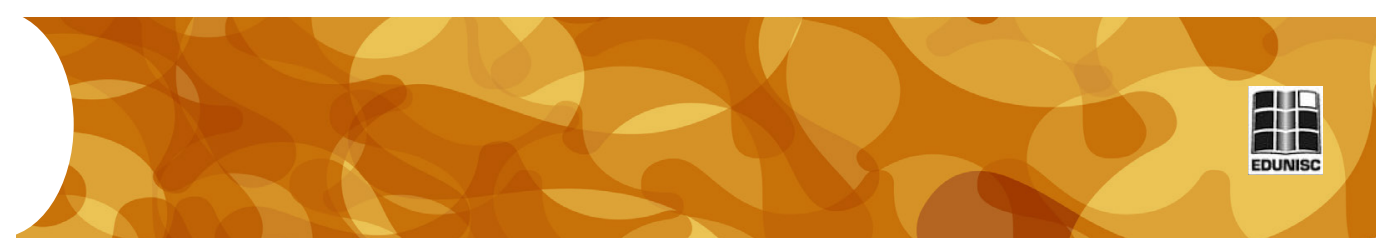

Toda circunscripción, sin embargo, parece encontrar sus límites. Gastón Cingolani, describe las acciones que se pueden aplicar dentro en cada plataforma musical (Spotify, Soundcloud, etc.): a. Buscar/Encontrar música; b. Reproducir (o "Escuchar") música; c. Cargar/descargar música; d. Organizar música; e. Compartir música (CINGOLANI, 2018, $\mathrm{s} / \mathrm{p}$ ). Una mirada rápida nos muestra que a., b. y d., son circulaciones internas a la plataforma de que se trate, pero c. y e. obligan a interactuar con el afuera de las plataformas. Esos recorridos entre el adentro y el afuera son muy importantes en el trabajo de Cingolani, y se volverá sobre ello más adelante.

\section{La circulación transmedia}

Hay otro tipo estudio, de muy extenso desarrollo, sobre el aporte de la circulación a secuencias o a estados de algún campo de la vida sociocultural y sus sistemas de mediatización. Ello se ve entre nosotros en una serie de investigaciones sobre la circulación de objetos artísticos.

Uno de los trabajos de interés en la búsqueda de relacionar a la circulación de la obra de arte con sus mediatizaciones es el artículo de Oscar Traversa, Sergio Ramos y Manuel Libenson acerca la construcción de valor de las obras en sistemas mediáticos que no sólo integran las de redes y plataformas.

Los autores sostienen que en esa circulación intervienen cuatro ejes: "1. La constitución de las relaciones productor - producto consumidor... 2. la puesta en serie de los objetos entre sí...3. La vigencia de un nivel metadiscursivo... que organiza espacios de intercambio y 4. La articulación de esas discursividades con la vida de los dispositivos técnicos..." (TRAVERSA et AL, 2014: s/p). Como se ve, esos ejes están relacionados en términos de circulación.

En el desarrollo de la ponencia, si bien los investigadores se refieren al nivel de la producción de sentido y a la construcción de valor de la obra de arte, utilizan tanto el término circuito como, cuando van hacia un nivel mayor de complejidad, el de circulación en un nivel más amplio, pero siempre manteniéndose en el nivel de la producción de sentido. Parecen sostener que no es lo mismo estudiar el recorrido de una obra de Goya, para tomar un ejemplo de ellos, que estudiar el fenómeno de la crítica como uno de los espacios que participa en todo el sistema. Esta oscilación, no sólo no les quita valor a las propuestas, sino que augura la posibilidad de adaptación a diferentes objetos de investigación en diversas etapas. Por otra parte, ese recorrido complejo y variado les lleva a confirmar la necesidad de mantener la distancia entre la producción y el reconocimiento veronianos. Es decir que, en esta aproximación, conviven previos y nuevos modelos de análisis e interpretación. 
Gastón Cingolani, por su parte, viene haciendo un recorrido sobre el terreno de los sistemas de recomendación en red (SR). El acceso a múltiples SR obliga a diferenciar entre sistemas humanos y maquinísticos, provenientes de algoritmos de robots y entre movimientos muy diferentes entre sí, como la distinción entre buscar y encontrar recomendaciones (CINGOLANI, 2017: 40-44).

Pero la propia problemática de las recomendaciones, aun dentro de plataformas musicales, lleva estas investigaciones a territorios extraplataformas. Entre diversas publicaciones que se van haciendo en la Web basados en big-data de Spotify, la página de Medium Corporation presenta a Sophia Ciocca como una "ingeniera en software" que va a explicar el lugar de “...la ciencia detrás de las recomendaciones personalizadas de música". El artículo de Ciocca explica los métodos de Spotify para construir una playlist semanal, denominada Discover Weekly que cada lunes presenta a usuarios premium una lista de 30 temas que cada uno de ellos "nunca ha escuchado pero que probablemente amen". Ciocca considera que nadie conoce mejor sus gustos que este servicio. El tema interesa porque es un especial cruce entre curaduría y archivos (FERNÁNDEZ, 2018b).

Según Ciocca, Spotify aplica tres modelos de recomendación diferentes, que no vamos a detallar aquí: filtrado colaborativo, a la manera de Netflix, pero incluyendo las solicitudes de cada tema; Natural Language Processing (NLP) models, softwares de análisis de textos, que seleccionan términos/etiquetas que aparecen como de agrado en los etiquetados y, como lo más novedoso, mediante y modelos de análisis de audios en bruto (Raw Audio Models), que bajo softwares como los de los detectores de rostro, permitirían seleccionar perfiles de sonido, aparentemente más allá de lo genérico-estilístico. Dejando de lado si estas son certezas o especulaciones de Ciocca, muestra que la producción y circulación de recomendaciones, está alcanzando una complejidad que nos saca de la comodidad de nuestros modelos de trabajo.

De estas observaciones se puede inferir que, además del modelo de secuencia y el de estado, habría que recordar el de circulación en ramificación: la observación de algunos fenómenos micro en plataformas nos lleva a territorios inesperados y muchas veces, inexplorados.

\section{La circulación en fases transmediáticas}

Hemos citado en la introducción el punto de vista macro de Mario Carlón con sus proposiciones que son de sumo interés porque articulan una temporalidad de fases, que son a su vez enfoques espaciales. Se trata de un típico efecto de postbroadcasting, al necesitar articular 
medios masivos con lo que él prefiere seguir denominando redes. En la Figura 4, se ve que en cada fase que, además de estar descripta de acuerdo a su posición en los diferentes sistemas mediáticos, se presupone un análisis aplicando el modelo, en principio triádico de Verón, que permite mantener la distancia, o al menos la oposición, entre producción y reconocimiento.

Figura 4 - Fases ascendentes y descendentes

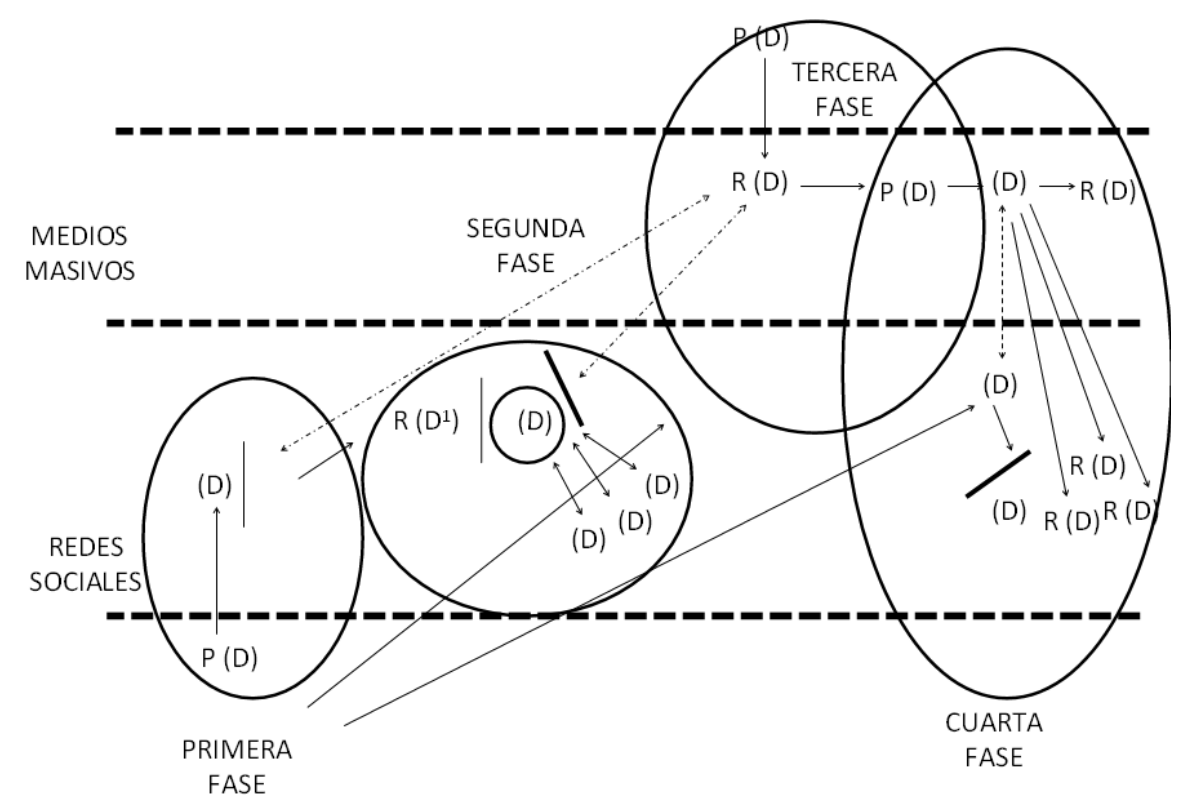

Respecto de la circulación como recorrido de construcción en red, se vienen publicando desde hace tiempo trabajos sobre cómo se relacionan diversos circuitos de lo musical, incluyendo cómo se relacionan entre sí diversos metadiscursos copresentes o diferentes en plataformas de lo musical (KOLDOBSKY, 2016).

Ese tipo de trabajo creemos que debería converger con experiencias de análisis como las que viene realizando el equipo de Carlón. Esas diferentes fases, en caso de que se vayan confirmando las conclusiones, permitirían comparar los procesos de desarrollo cultural de la actualidad con los de épocas de broadcasting a los que tendemos ahora también a ver con aspectos reticulares y con juegos de plataformas. Se ha encontrado que el despliegue del tango canción, por ejemplo, aparece como descendente desde los medios hacia el espacio social masivo (JÁUREGUI, 2013). En cambio, el folklore tiene un largo recorrido en los teatros de las redes ferroviarias hasta consolidarse en los medios (ascendente) (FERNÁNDEZ, 2018: 56-60).

Pero, además, por un lado, está el estudio de fases en secuencias, que son los ejemplos que hasta ahora ha desarrollado el equipo de Carlón, que se encuentran en circuitos (otra vez el término), como el de la moda, 


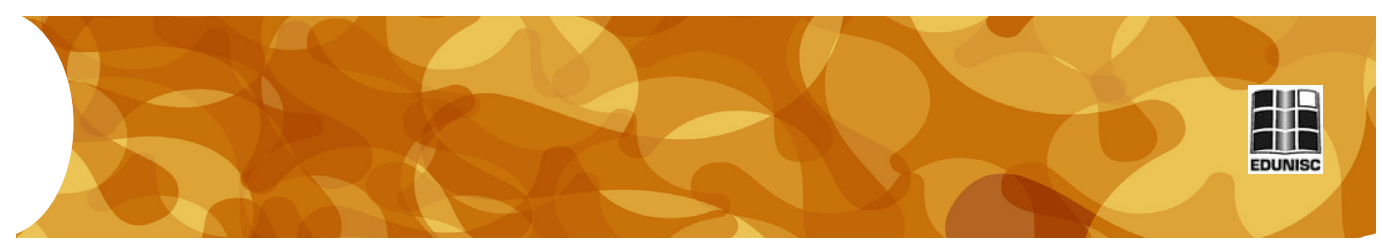

sitios específicos como los blogs especializados (GÓMEZ DEL RÍO, 2017: s/p). Los blogs de moda, perfectamente estabilizados, funcionan como fase, cuando se lanza una nueva temporada o colección, pero son estados si se quiere comprender el funcionamiento de la moda.

\section{La circulación en fases transmedia-cara a cara}

Por último, nos vamos a referir a los casos muy importantes en las vidas de lo musical, pero no exclusivos de ellas (el de la moda, los lugares de comida, los eventos de diverso tipo, funcionan de un modo parecido), en las que performances en vivo conviven con modos de intervención o difusión mediáticas, a través de plataformas generales o propias.

Ya cuando periodizamos relaciones entre mediatizaciones y músicas, nos vimos obligados aincluir, para cada período, las modalidades más importantes de las performances cara a cara (FERNÁNDEZ, 2013: 36-40). Así, por ejemplo, la expansión de lo digital y las plataformas musicales, convive con las Raves, mientras permanecen los grandes conciertos con despliegue audiovisual explosivo, pero al mismo tiempo, se desarrollan diversos circuitos alternativos de centros culturales sostenibles, con fuerte comunicación en redes.

Uno de los casos de relaciones entre espacios públicos y mediatizaciones que se han descripto, es el de los sonideros, dj's callejeros de México D. F. con su público y, a través de ellos, de su público con familiares o redes. Rubén López-Cano lo describe así:

\footnotetext{
La audiencia le entrega todo tipo de mensajes al sonidero para que los lea durante el show. Estos saludos son para familias y amigos, barrios, clubes de baile o simplemente anuncios de que alguien en particular está presente en el espectáculo. Gente famosa o bailarines solo van a la cabina de DJ para anunciarse (LÓPEZ-CANO, 2015: 148).
}

Otro caso de relación entre espacio público, de sentido inverso al anterior, es el de los flashmob que

\footnotetext{
son posibles gracias al uso de las nuevas tecnologías masificadas, y define una acción colectiva organizada en un lugar público, inusual, donde participa un grupo numeroso de individuos que al terminar se dispersan inmediatamente (CID JURADO, 2015: 46).
}

Como se dijo al principio, la noción de circuito es clave para comprender el funcionamiento de lo musical según géneros o estilos musicales, que se van construyendo de formas específicas, según características, tanto musicales como socioculturales. Amparo Rocha Alonso describe así los parecidos y diferencias entre el circuito del 


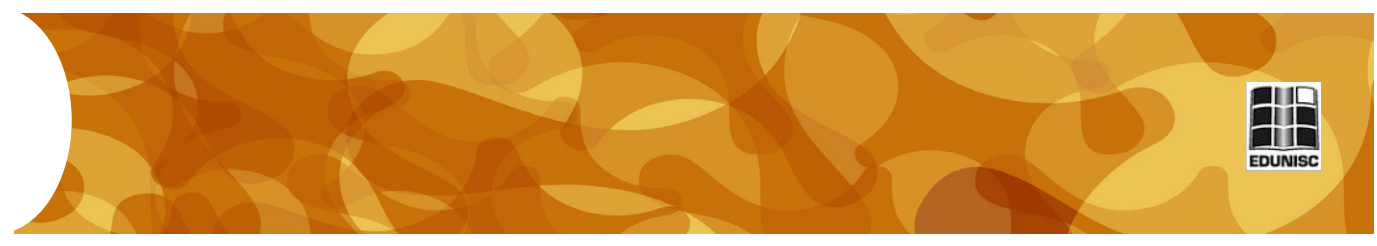

freestyle y lo que denomina música popular de cámara, que hunde sus raíces en la tradición de cierta música considerada críticamente como folklórica y de raíz latinoamericana:

\begin{abstract}
El circuito de freestyle va de la crudeza de los cuerpos actuantes en la interacción cara a cara a la crudeza de las grabaciones caseras que se suben a Internet. El circuito de las músicas populares de concierto apunta doblemente al vivo, bajo ciertas condiciones básicas de calidad (buen sonido, público en posición de escucha, eventual escenografía artística) y a la grabación, tanto en formato material $\mathrm{CD}$, como en plataformas como Bandcamp, Soundcloud, Vimeo o Youtube.
\end{abstract}

Pero esa segmentación en los modos y las materialidades de mediatización se produce dentro mismo, por ejemplo, del freestyle. Mariano Lapuente, que ya había publicado un trabajo sobre las relaciones entre conciertos en vivo y su publicación en YouTube, en un informe reciente de investigación, basado en experiencias realizadas con alumnos dentro de la Cátedra Semiótica de las mediatizaciones, diferencia dos casos fuertes y muy diferentes dentro del freestyle:

(P)or un lado las batallas de rap improvisado en el marco del Quinto Escalón que es una comunidad que hasta el 2017 se reunía en el Parque Rivadavia de la Ciudad Autónoma de Buenos Aires, y por otro, la Batalla de los Gallos que organiza la marca Red Bull a nivel nacional e internacional (LAPUENTE, 2018, s/p).

Sólo con esa primera descripción, enriquecida en el conjunto del trabajo con un muy bien aplicado modelo peirceano, da una idea sobre las relaciones entre el arriba y el abajo, el adentro y el afuera, y los formatos de circuitos o de ramificación. La experiencia de surgimiento, éxito y masificación, hasta la decisión de sus responsables de cerrarla en el 2017, es narrada hoy en las redes como circuito.

\title{
8. Síntesis provisoria
}

Esta síntesis no puede ser sino provisoria por lo acotado del recorrido bibliográfico, pero, al menos, se han observados diversos tipos de enfoques que enfrentan muchos de los problemas claves de la circulación de las mediatizaciones en la actualidad.

Un recorrido de sobrevuelo por el contenido de este artículo muestra que la circulación es un tema que seguirá siendo importante. La discusión, si la hay, será en todo caso si se deben incluir todos los fenómenos descriptos aquí dentro del paraguas macro de la circulación.

Al avanzar en el estudio de fenómenos de circulación ocurre lo 


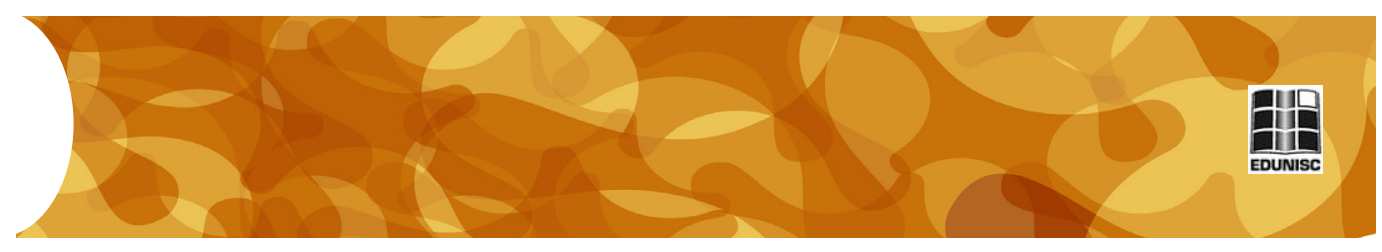

mismo que cuando se estudian otros fenómenos sociales desde un punto de vista micro: lo que se observa como coherente e integrado desde un punto de vista general, macro, tiende a disolver sus certezas frente a la aparición de rasgos novedosos y al aportar a series inesperadas del campo de estudio.

Queda la inquietud acerca de si el estudio de la circulación, luego de este momento refundacional, se constituirá en un campo de vida independiente, con metodologías propias. Parecen afirmar esa perspectiva de la circulación como fenómeno macro, tanto las proposiciones de Antonio Fausto Neto, sobre las nuevas arquitecturas mediáticas, como las de Mario Carlón, acerca de que se está frente a uno de los rasgos claves que constituyen la contemporaneidad. Pensadas así, esas formulaciones resultan indiscutibles. Pero queda la duda de que, si cuando se estudia un caso particular, se debería comenzar por esos nuevos conceptos generales o se debería, en cambio, derivar a través de lo que se va encontrando en el camino de observación y grillado de las investigaciones. La recuperación parcial de la noción de circuito muestra que el camino no es lineal.

Además, ¿cómo hacer para incorporar las relaciones entre espacios cara a cara y diversos tipos de mediatización? ¿Se los dejará afuera por no ser mediáticas las performances en vivo y cara a cara? ¿O habrá que agregar ese nivel al de los medios masivos y las plataformas o redes? ¿Y las mediatizaciones en las performances en vivo?

Por último, es necesario reivindicar la importancia que tiene la sociosemiótica de los intercambios e interacciones discursivas para la comprensión de los diversos tipos de circulación. Como en otros niveles de aplicación teórica, se nota aquí esa característica que hace que sea posible ir de lo discursivo hacia un hábito social, mientras que es imposible el camino inverso: nunca desde un hábito se irá hacia la construcción del sentido de los intercambios e interacciones en las redes. Las plataformas mediáticas son, desde este punto de vista, el espacio en que esos juegos de interfaz son posibles y en cuyas discursividades se encuentran marcas de los intercambios.

\section{Referencias}

ARUGUETE, Natalia y CALVO, Ernesto. "El patrullaje anónimo. Campañas de trolls y fakes". En: Revista Anfibia, Universidad Nacional de San Martín. En: http://revistaanfibia.com/ensayo/elpatrullaje-anonimo/. Última revisión: 08/12/2018. 


\section{Rizoma}

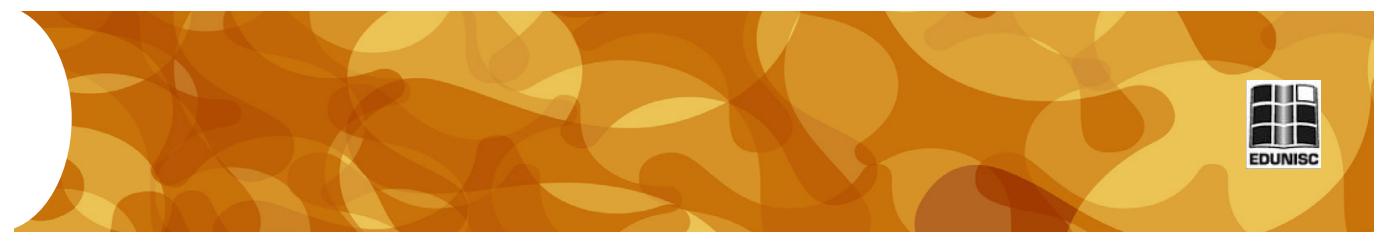

BRAGA, Jose Luis. "La política de los internautas es producir circuitos". En: CARLÓN, Mario y FAUSTO NETO, Antonio. (Comps.) La política de los internautas. Nuevas formas de participación. Buenos Aires: La Crujía, 2012. pp. 43-59.

CARLÓN, Mario. "Medios individuales, medios colectivos y circulación transversal. Desde "adentro hacia afuera" y desde "afuera hacia adentro" (o como afecta la nueva circulación a las instituciones sociales). En: CASTRO, P. C. (Organizador) Circulação discursiva e transformação da sociedade. Campina Grande-PB: Eduepb, 2018. (s/p).

CARLÓN, Mario, FRATICELLI, Damián, SLIMOVICH, Ana. Documento teórico metodológico. Cátedra semiótica de redes. Buenos Aires: Carrera de Ciencias de la Comunicación. Facultad de Ciencias Sociales - Universidad de Buenos Aires, 2016.

CID JURADO, Alfredo Tenoch. "Visión espectacular, visión mediatizada en el caso del flashmob". En: Letra. Imagen. Sonido L.I.S. Ciudad Mediatizada. Buenos Aires: UBACyT, 2011. No 10, 1do. Semestre 2015, pp. 43-60.

CINGOLANI, Gastón. "Sistemas de recomendación. Mediatizaciones con rebordes enunciativos de los juicios de gusto". En Foro: ESTILOS Y MEDIATIZACIONES Buenos Aires: Instituto de Investigación y Experimentación en Arte y Crítica, UNA, s/f.

CINGOLANI, Gastón. "Sistemas de recomendación, mediatizaciones de lo preferible y enunciación”. En: BUSSO, Mariana y CAMUSSO, Mariángeles (Eds.) Mediatizaciones en tensión : el atravesamiento de lo público. Rosario: UNR Editora, 2017, pp.30-47.

CINGOLANI, Gastón. "Circulación y mediatización de la experiencia estética”. En: CASTRO, P. C. (Organizador) Circulação discursiva e transformação da sociedade. Campina Grande-PB: Eduepb, 2018. (s/p).

CIOCCA, Sophia. How Does Spotify Know You So Well? En Medium Corporation. https://medium.com/s/story/spotifys-discover-weeklyhow-machine-learning-finds-your-new-music-19a41ab76efe, 2017 Última consulta 02-06-2018

FAUSTO NETO, Antonio. A circulaçao além das borda. En: En: Neto, F. y Valdettaro, S. Mediatización, Sociedad y Sentido. Rosario: UNR 


\section{Rizoma}

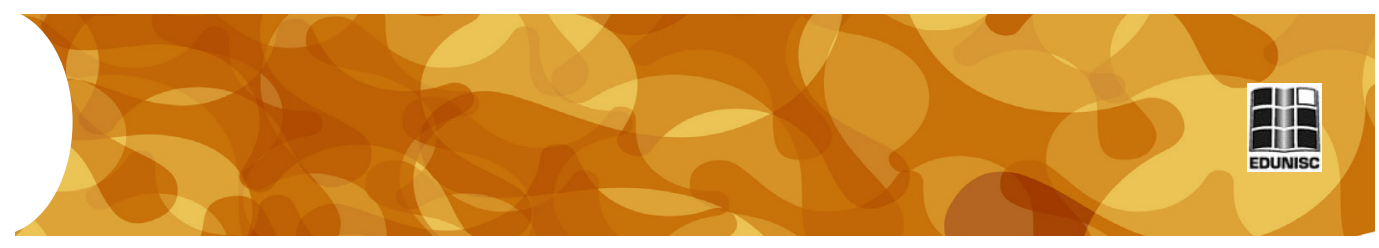

Editora, Agosto 2010 pp. 2-17.

FERNÁNDEZ, José Luis. "Hacia los efectos de lo radiofónico". Parte III de La captura de la audiencia radiofónica. Buenos Aires, Líber Editores, 2012 pp. 239-279.

FERNÁNDEZ, José Luis. "Interacción: un campo de desempeño múltiple en broadcasting y en networking". En: Cingolani, G., Sznaider, B. (eds.). Cuadernos del CIM. Rosario: UNR Editora. Pp. 10-26, 2016.

FERNÁNDEZ, José Luis. Plataformas mediáticas. Elementos de análisis y diseño de nuevas experiencias. Buenos Aires: La Crujía, 2018a.

FERNÁNDEZ, José Luis. "Semiótica, ecosistemas y big data en la captura de los usuarios de plataformas de música". Presentado en el XIII Congreso de(IASPM-AL. San Juan, Puerto Rico, junio de 2018b (en prensa).

FERNÁNDEZ, José Luis. y JÁUREGUI, Jimena. "El networking musical porteño: el tango en la ciudad mediatizada". En: VARGAS, H y KARAM, T (Eds.) De norte a sur: música popular y) ciudades en América Latina. Apropiaciones, subjetividades y reconfiguraciones. Mérida: Consejo Nacional para la Cultura y las Artes, 2015, pp. 413-440.

GÓMEZ DEL RÍO, Gabriela. Los cibergéneros especializados: gestión de contenidos en weblogs independientes de moda. Buenos Aires: UBA. Facultad de Ciencias Sociales - Maestría en Periodismo, 2017.

JÁUREGUI, Jimena Anabel, “Tango y gran ciudad. La sonoridad gráfica del tango, Dossier Elementos para la conformación del broadcasting musical - Letra. Imagen. Sonido. L.I.S. Ciudad Mediatizada, \#9, 2013, pp. 114-127.

LAPUENTE, Mariano. Los conciertos en vivo y su procesamiento en las redes: la experiencia social de la música en YouTube. En: Fernández, J. L. (Coordinador). Postbroadcasting. Innovación en la industria musical. Buenos Aires: La Crujía, 2013.

LAPUENTE, Mariano. "El vínculo social en la música entre el espacio social y el espacio mediatizado". Informe interno en el Proyecto 


\section{Rizoma}

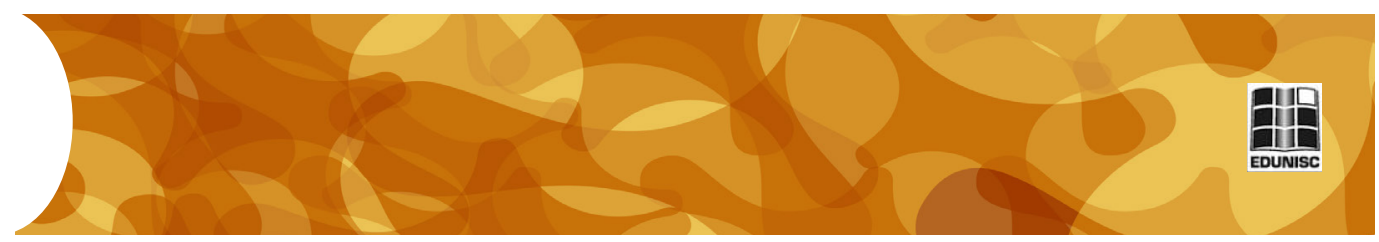

"Letra, Imagen, sonido." (UBACyT - 2014-2017), Buenos Aires: 2018. (inédito)

LÓPEZ-CANO, Rubén. "Mexican Sonideros: alternative bodies on streets". En: INMEDIACIONES DE LA COMUNICACIÓN, - VOL. 10 - N ${ }^{\circ} 10$. Montevideo, Escuela de Comunicación, ORT, pp. 145-155.

NETO, Antonio. F. A circulaçao além das borda. En: En: Neto, F. y Valdettaro, S. Mediatización, Sociedad y Sentido. Departamento de Ciencias de la Comunicación. Facultad de Ciencia Política y RRII. UNR. Rosario - Agosto 2010.

PORTO LÓPEZ, Pablo. La escritura del directo. Transformaciones y permanencias de lo radiofónico en las transmisiones deportivas de los diarios en Internet. En: Letra. Imagen. Sonido L.I.S. Ciudad Mediatizada \#5 Buenos Aires: UBACyT, 2010. Pp. 107-125.

PORTO LÓPEZ, Pablo. "Minuto a minuto: ¿El live blogging como nueva instancia de construcción del acontecimiento en directo? En: L.I.S. Ciudad Mediatizada \#11 Buenos Aires: UBACyT, 2010. Pp. $127-150$

PORTO LÓPEZ, Pablo. Tiempo, escritura y medios digitales. La crónica simultánea y la construcción del acontecimiento en desarrollo a través de lo escrito. Tesis de Maestría en Análisis del Discurso. FFyL-UBA, 2018 (no publicada).

ROCHA ALONSO, Amparo. 'Proyecto burbujas: circuitos de música en Buenos Aires’, L.I.S. \# 15 (2016), pp. 35-48.

SCOLARI, Carlos. A. Ecología de los medios: de la metáfora a la teoría (y más allá). En: Scolari, C. A. (Ed.) Ecología de los medios. Entornos, evoluciones e interpretaciones. Barcelona: Gedisa, 2015, pp. 15-42.

THURMAN, Neil y WALTERS, Anna. "Live Blogging- Digital Journalism's Pivotal Platform". Digital Journalism 1.1, 2013, pp. 82101.

TRAVERSA, Oscar. La fatalidad de la calle. En: L.I.S. \#1. Buenos Aires: UBACyT, 1er. Semestre, 2015.

TRAVERSA, Oscar. Aproximaciones a la circulación a partir de Eliseo Verón, Conferencia pronunciada en el Centro Internacional de 


\section{Rizoma}

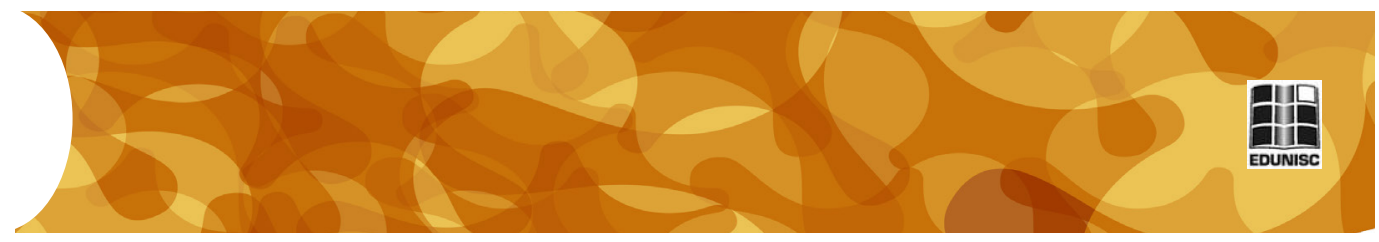

Semiótica y Comunicación (CISECO), PENTALOGO VII, el 21 de setiembre de 2016, en Japaratinga, Alagoas, Brasil, 2016.

TRAVERSA, Oscar; RAMOS, Sergio y LIBENSON, Manuel. Valor y circulación discursiva: el caso del arte. En XVIII Jornadas Nacionales de Investigaciones en Comunicación. Buenos Aires: REDCOM-UNA, octubre de 2014. s/p.

VERÓN, Eliseo. Prefacio a la segunda edición. En: Construir el acontecimiento. Barcelona: Gedisa, 1987. 